\title{
Application of a Quartz Crystal Microbalance (QCM) System Coated with Chromatographic Adsorbents for the Detection of Olive Oil Volatile Compounds
}

\author{
María E. Escuderos ${ }^{1 *}$, Sebastián Sánchez ${ }^{2}$, Antonio Jiménez ${ }^{1}$ \\ ${ }^{1}$ IFAPA Venta del Llano (Andalusia Regional Government), Jaén, Spain \\ ${ }^{2}$ Department of Chemical, Environmental and Materials Engineering, University of Jaén, Jaén, Spain \\ E-mail:mariae.escuderos@hotmail.com,ssanchez@ujaen.es,antonio.jimenez.marquez@juntadeandalucia.es \\ Received January 7, 2011; revised March 7, 2011; accepted March 9, 2011
}

\begin{abstract}
A sensor based on the technique of a piezoelectric quartz crystal microbalance (QCM) is analyzed for the detection of six organic volatile compounds with high olive oil sensory significance, such as hexanal, acetic acid, Z-3-hexenyl acetate, undecane, 1-octen-3-ol and 2-butanone. Four sample concentrations have been exposed to each QCM sensor constructed. The detection system is based on the sample adsorption on the forty sensing films coated at the surfaces of forty AT-cut gold-coated quartz crystals. Each sensing film has been prepared with different solution concentrations of ten materials, usually used as chromatographic stationary phases. Sensing film coating process shows excellent repeatability, with coefficient values less than $0.50 \%$. The frequency shifts of the piezoelectric crystals due to the adsorption of the volatile compounds have been measured as sensor responses, using a static measurement system. The results show that only five QCM sensors, with high sensitivity values, are enough to the detection of the volatile compounds studied. Therefore, the developed detection system presented herein provides a rapid identification of organic volatile compounds with elevated olive oil sensory connotation and it could be a substitute technique to the analytical methods normally used for the analysis of the olive oil flavor.
\end{abstract}

Keywords: QCM Gas Sensor, Chromatographic Adsorbents, Olive Oil, Volatile Compounds, Sensory Connotations, Electronic Nose

\section{Introduction}

Sensory evaluation of virgin olive oil is standardized by a European Union regulation [1] and trade standards of the International Olive Oil Council [2]. Panels of trained assessors are used in both cases to evaluate a set of sensory descriptors. Sensory descriptors of virgin olive oil can be classified into "positive" and "negative". The latter describes defects of virgin olive oil and they are mainly fusty, musty, muddy sediment, vinegary, metallic and rancid. These defects are mostly explained by volatile compounds, which are produced by biogenic pathways of olives, over-ripening of the olive fruit, oxidation of the unsaturated fatty acids and attack by moulds and bacteria by an inappropriate olive harvesting process and by inadequate olive paste manipulation during the olive oil extraction process in the olive oil mill [3].
The identification of olive oil defects is usually carried out by two procedures: the analysis of the volatile compounds and the sensory panel test [4]. The first one is an increasingly used technique in the characterization of food products. The analytical methods normally used require different extraction and chromatographic techniques, in particular, gas chromatography coupled to mass spectrometry. Normally, the steps involved in chromatographic separation and identification require too much time to be used routinely in the food industry, so it cannot be applied on-line in the processes of storage and bottling of virgin olive oil.

The official method to olive oil sensory evaluation is panel test, which is regulated by Regulation EC/640/ 2008 [1]. According to its criteria set, a virgin olive oil is graded on the basis of fruitiness and intensity of defects (defined previously) by a group of tasters operating. A 
panel test consists of a panel head and from eight to twelve tasters. The panel head must be a soundly trained expert in the various types of oil and tasters must be selected and trained on account of their skill in distinguishing between similar samples. The panel test must follow the International Olive Oil Council's manual on the selection, training and monitoring of qualified virgin oil tasters [2]. According to this manual, it is recommended that a taster evaluates twelve samples by day, divided in three sessions, to avoid the exhaustion of the senses of smell and taste. So, organoleptic assessment of an olive oil sample, by the panel test methodology, has the great disadvantage of being a lengthy and expensive methodology whose final result depends on many factors, such as the panelists' training.

An alternative technique to analyze olive oil flavor is based on the use of chemosensors for detecting volatile compounds present in the headspace of foodstuffs. Chemosensors have been widely adopted in the quality assessment of foodstuffs, such as wine, apple, grape, banana, pear, peach, milk, yoghurt, tomato, water, cheese, sugar, chocolate, fish, Iberian jam and vegetable oils [5].

Among all kinds of sensors, quartz crystal microbalance (QCM) sensor has attracted our attention for its low cost, compact volume, easy portability and high sensitivity [6]. QCM is extensively employed in gas analysis since it was first introduced by King in 1964: detection and identification of organic vapors, food analysis, continuous monitoring of volatile chloroorganic compounds, determination of iodine in foodstuffs, QCM as humidity sensor, detection of BTEX compounds vapors, speciation of nitroaromatic compounds in landfill gas, detection of wine volatiles, determination of organophosphorus and carbamate pesticides, monitoring of apple flavor, determination of the ripening state of Emmental cheese, monitoring of the performance of an odor biofilter, characterization of Fuji apples, determination of ammonia and aliphatic amines, rapid detection of Escherichia coli O157:H7, determination of chlorinated aliphatic hydrocarbons in air, Salmonella detection, tomato aroma profiling, discrimination of $\mathrm{CH}_{3} \mathrm{SH}$, identification of volatile organic compounds, detection of mesothelin in pancreatic cancer cell line supernatant, GMOs detection, determination of trace metal ions in solution or detection of microbial populations $[5,7,8]$.

In this work, we have studied the potential of an artificial olfactory system, constituted by quartz crystal microbalance sensors, to detect organic volatile compounds with a high olive oil sensory significance, as an alternative to the difficult and time-consuming analytical methods described previously. Therefore, ten chromatographic stationary phases were coated onto forty quartz crystal surfaces as sensing films, to study and select those who best respond and discriminate six organic volatile compounds responsible of different sensorial attributes of olive oil flavor.

\section{Experimental}

\subsection{Materials}

According to previous papers $[9,10]$, six organic volatile compounds with high olive oil sensory significance have been selected: hexane, Z-3-hexenyl acetate and 2-butanone as green notes; 1-octen-3-ol, as responsible for musty-humid attribute; acetic acid, which is the volatile compound more contributes to winery-vinegary olive oil defect; and undecane, as neutral volatile. The six volatile compounds were purchased from Sigma Chemical Co. (St Louis, USA) and utilized without further purification.

Ten gas chromatographic stationary phases were selected and used as sensing films due to their characteristics of polarity, stability and sensibility [11]: $O V-101$, Vaseline, Di-n-onyl phthalate (DNP), Span 85, OV-275 and Polyethylene glycol (PEG) were purchased from Fluka Co. (Buchs, Switzerland); OV-17 and Carbowax $20 M$ were obtained from Supelco Inc. (Bellefonte PA, USA); Polydimethylsiloxane and Span 80 were purchased from Sigma Chemical Co. (St Louis, USA). Polyvinyl chloride $(P V C)$ with high molecular weight was purchased from Fluka Co. (Buchs, Switzerland) and Tetrahidrofurane (THF) was obtained from Supelco Inc. (Bellefonte PA, USA).

A spin coating method was used to coat the adsorbent on the surface of the gold electrodes [12]. An adsorbent and PVC were mixed together and dissolved in THF [13]. Ten microliters of the cocktail were dispensed onto the surface of the gold electrode by using a micropipette. The Tetrahidrofurane dries quickly leaving a thin film of adsorbent on the surface of the quartz crystal. This process guarantees applying a reproducible amount of coating material onto a quartz crystal.

\subsection{Instrumentation}

The centrifuge SPIN 150 Wafer Spinner, from KSV Company (Helsinki, Finland), was used to coat the sensing film onto the quartz crystals. The QCM system used consists of the QCM100 Analog Controller, QCM25 Crystal Oscillator, QCM Crystal Holder and the QCM Crystal (Stanford Research System Company, Sunnyvale, California). This system comprises a 5-MHz AT-cut quartz resonator with gold electrodes as a QCM gas sensor. Sensors results were stored in a personal computer through a multi-channel frequency counter. 


\subsection{Experiment Protocol}

In the present work, a steady-state measurement system was used to evaluate sensing films characteristics of QCM gas sensors. The schematic diagram of the system is shown in Figure 1 [5,14]. The liquid sample injected in the measurement chamber $\left(500 \mathrm{~cm}^{3}\right)$ was kept at a temperature of $28 \pm 2^{\circ} \mathrm{C}$, according to the IOOC regulation [2]. The amount of sample $\left(5 \mathrm{~mm}^{3}\right)$ was injected 4 times (each 600 seconds) to correlate the volatile compound concentrations and the sensor responses; i.e. sample concentrations inside the measurement chamber were $10,20,30$ and $40 \mathrm{ppm}$ at $0,600,1200$ and 1800 seconds. Frequency values were measured each 2 seconds. As an example, Figure 2 shows the in-process frequency drop of sensor S28 (PEG adsorbent) exposed to increasing concentrations of acetic acid volatile compound. At the equilibrium point, a sensor response was defined as the

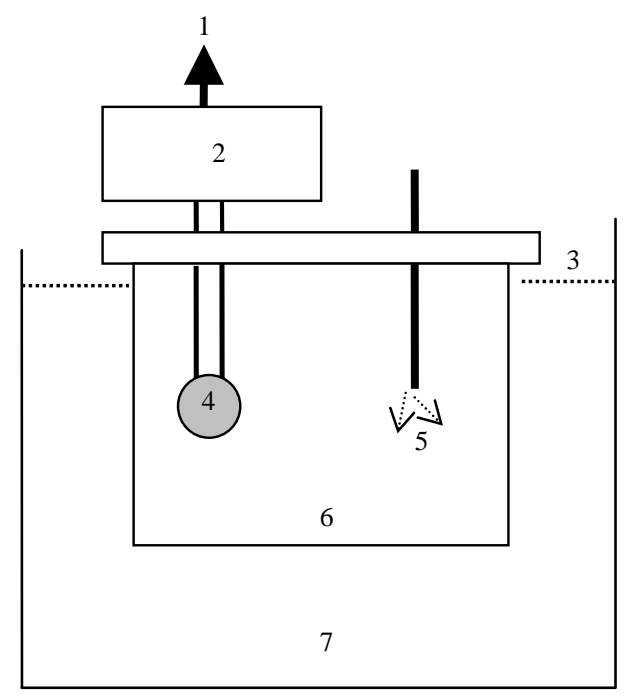

Figure 1. Schematic diagram of measurement system [(1) data analysis, (2) oscillation circuit, (3) water level, (4) QCM gas sensor, (5) sample injection, (6) measurement chamber, (7) thermostatic bath].

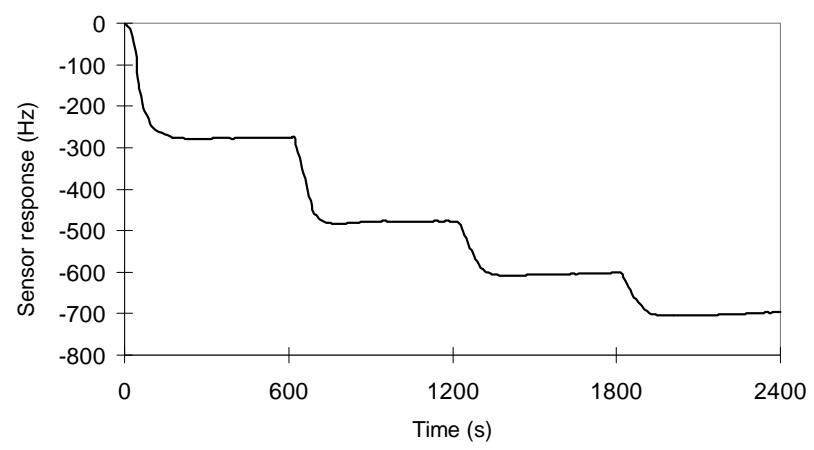

Figure 2. Response curve ( $\mathrm{Hz})$ of sensor S28 (PEG) to 5 $\mathrm{mm} 3$ of acetic acid injection at $0,600,1200$ and $1800 \mathrm{sec}-$ onds. shift of the oscillation frequency of the QCM connected to an oscillator.

\section{Results and Discussion}

\subsection{Sensor Construction Process Reproducibility}

Sensor construction process repeatability was studied by using OV-275 adsorbent chromatographic material because it is easier to apply due to its viscosity. Therefore, sensing film of sensor $S 24$ (6 mg OV-275 adsorbent in a tetrahidrofurane solvent solution [6]) was prepared and dropped, by spin coating method (Subsection 2.1), onto the gold electrode surface of the same quartz crystal resonator, during five days and three times a day, i.e. sensor $S 24$ was constructed fifteen times. The base frequency value of the quartz crystal resonator used was $5007.511 \mathrm{kHz}$. At the equilibrium point, frequency oscillation value of each quartz crystal resonator, with the coated sensing film, was registered.

The relative standard deviation (SD) and variation coefficient (CV) were the parameters considered (Table 1) to analyze the sensor construction repeatability. The inter-day and intra-day reproducibility values are less than $0.50 \%$, so the sensing films coating process suggested in this work is very good and guarantees applying a reproducible amount of coating material onto a quartz crystal resonator.

\subsection{Sensors Selection by Sauerbrey Equation}

QCM sensor oscillation frequency, $\Delta F(\mathrm{~Hz})$, is linearity related to quartz crystal mass change $(\Delta m)$ due to Sauerbrey equation [12]:

$$
\Delta F=-C_{f} \cdot \Delta m
$$

Four amounts of each chromatographic adsorbent were studied to prepare forty sensing films. They were constituted by 1, 2, 3 or $6 \mathrm{mg}$ of an adsorbent dissolved in $1 \mathrm{ml}$ of PVC/THF solution. Each sensing solution was coated onto the surface of each quartz crystal to construct forty QCM gas sensors. Sensors oscillation frequency shifts $(\mathrm{Hz})$, due to sensing films coating process, are shown in Table 2. In general, sensor construction process produces small mass increases $(\Delta m)$ onto the surface of the quartz crystals, so it doesn't reduce adsorption capacity of the QCM gas sensors. However, according to $\mathrm{Ni}$ et al. criterion [11], a QCM gas sensor response can be explained by the Sauerbrey equation when its sensing film coating process produces a frequency change $(\Delta F)$ -that is the shift on the oscillating frequency of the QCM between before and after sensing film coating statesamong 3 to $9 \mathrm{~Hz}$. Therefore, only the following eight 
Table 1. Repeatability measurements of $S 24(\mathrm{OV}-275)$ sensor construction process.

\begin{tabular}{ccccccc}
\hline Day & $1^{\text {st }}(\mathrm{kHz})$ & $2^{\text {nd }}(\mathrm{kHz})$ & $3^{\text {rd }}(\mathrm{kHz})$ & Dairy average $(\mathrm{Hz})$ & SD & VC $(\%)$ \\
\hline 1 & 5007.507 & 5007.506 & 5007.508 & 507.00 & 1.00 & 0.20 \\
2 & 5007.505 & 5007.509 & 5007.509 & 507.67 & 2.31 & 0.45 \\
3 & 5007.508 & 5007.506 & 5007.507 & 507.00 & 1.00 & 0.20 \\
4 & 5007.509 & 5007.507 & 5007.507 & 507.67 & 1.15 & 0.23 \\
5 & 5007.508 & 5007.504 & 5007.506 & 506.00 & 2.00 & 0.40 \\
Time average (Hz) & 507.40 & 506.40 & 507.40 & 507.07 & & \\
SD & 1.52 & 1.82 & 1.14 & 1.49 & & \\
VC (\%) & 0.30 & 0.36 & 0.22 & 0.29 & & \\
\hline
\end{tabular}

Table 2. Sensor oscillation frequency shifts due to sensing film coating process.

\begin{tabular}{ccccccc}
\hline \multirow{2}{*}{ Adsorbent } & \multirow{2}{*}{ Relative polarity $^{\mathrm{a}}$} & Sensor numbers & \multicolumn{3}{c}{$-\Delta \mathrm{F}(\mathrm{Hz})$} \\
\cline { 4 - 7 } Carbowax 20M & 3 & S01 to S04 & 1.096 & 1.693 & 2.794 & 4.278 \\
DMPS & 1 & S05 to S08 & 1.428 & 1.459 & 1.593 & 2.122 \\
DNP & 2 & S09 to S12 & 0.776 & 0.870 & 1.220 & 1.596 \\
OV-101 & 1 & S13 to S16 & 0.291 & 1.993 & 2.764 & 4.491 \\
OV-17 & 2 & S17 to S20 & 0.694 & 1.069 & 2.084 & 4.221 \\
OV-275 & 4 & S21 to S24 & 0.375 & 1.859 & 2.850 & 4.830 \\
PEG & 4 & S25 to S28 & 0.501 & 1.331 & 3.216 & 6.988 \\
SPAN 80 & 3 & S29 to S32 & 0.123 & 0.500 & 1.458 & 3.584 \\
SPAN 85 & 3 & S33 to S36 & 0.547 & 0.915 & 1.316 & 1.899 \\
Vaseline & 1 & S37 to S40 & 0.921 & 1.569 & 2.832 & 4.570 \\
\hline
\end{tabular}

a Information obtained from Ni et al., 2003; ${ }^{\mathrm{b}}$ Amount of adsorbent (mg) added to $1 \mathrm{ml} \mathrm{PVC/THF} \mathrm{solution.}$

sensors $S 4$ (Carbowax 20M), S16 (OV-101), $S 20$ (OV-17), S24 (OV-275), S27 (PEG), S28 (PEG), S32 (Span 80) and $S 40$ (Vaseline) can be used, because their performances can be justified by the Sauerbrey equation.

\subsection{Sensors Selection by Their Responses to Organic Volatile Compounds}

The eight sensors selected were exposed to different concentrations (10, 20, 30 and $40 \mathrm{ppm})$ of the six organic volatile compounds defined in Subsection 2.1.; $5 \mathrm{~mm}^{3}$ of each organic volatile compound was injected 4 times (each 600 seconds) in the measurement chamber, which had a volume capacity of $500 \mathrm{~cm}^{3}$. Due to Sauerbrey equation [12], a mass increase on the surface of the quartz resonator means a frequency reduction, so sensor responses are negative values.

Results from these experiments are summarized in Figure 3. Generally, QCM sensors respond linearity to the concentration of the analytes. It is clear that each QCM sensor, coated with a different sensing film, has quite different response intensities towards the same analyte, e.g. at a concentration of acetic acid inside the measurement chamber about 40 ppm, response intensity for each sensor decreases in the sequence of $S 28$ (PEG) > $S 24(\mathrm{OV}-275)>S 40$ (Vaseline) $>S 32($ Span 80$)>S 27$ $(\mathrm{PEG})>S 20$ (OV-17). Anyway, $S 4$ (Carbowax 20M), Figure 3(g), and $S 16$ (OV-101), Figure 3(a), get the saturation for acetic acid when the volatile concentration arrives 20 and 30 ppm, respectively. The array shows different response pattern towards other analytes, e.g. at a concentration of $40 \mathrm{ppm}$ of hexanal, sensors responses decrease in the following order: $S 32$ (Span 80) $>S 40$ (Vaseline) $>S 4($ Carbowax 20M) $>S 24(\mathrm{OV}-275)>S 20$ $(\mathrm{OV}-17)>S 27(\mathrm{PEG})>S 16(\mathrm{OV}-101)>S 28$ (PEG). On the other hand, one sensor presents different discrimination capacity to different organic volatile compounds (VOCs), e.g. response intensity decreases in order of Z-3-hexenyl acetate $>$ undecane $>$ hexanal $>$ 1-octen-3-ol $>$ acetic acid $>2$-butanone on sensor $S 24$ coated with OV-275 adsorbent (Figure 3(c)). Sensors $S 27$ and $S 28$ (Figures 3(e) and 3(f)), coated with 3 and $6 \mathrm{mg}$ of PEG adsorbent, respectively, have the same volatile compounds discrimination capacity, although $S 28$ responses are higher than the other one. Figure 3c shows that $S 32$, coated with Span 80, is useful to classify 1-octen-3-ol volatile compound, which is responsible of musty-humid olive oil attribute [9], due to its high response intensity to 


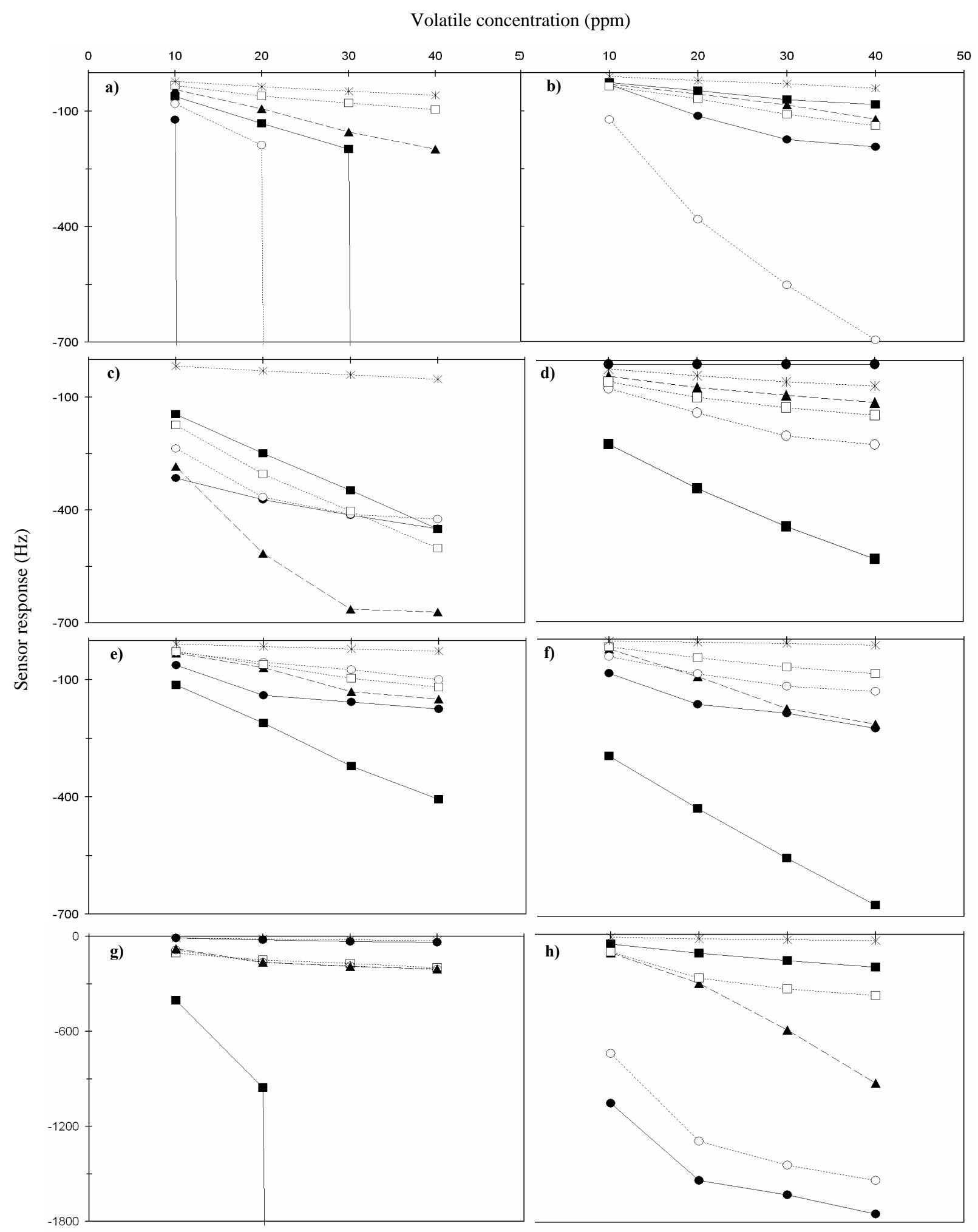

Figure 3. Relationships between organic volatile compounds concentrations $[(\bullet)$ undecane, $(\varpi)$ acetic acid, $(\circ)$ Z-3-hexenyl

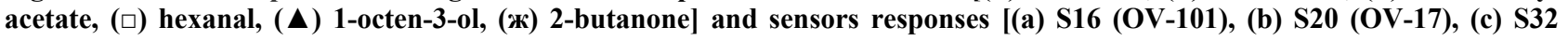
(Span 80), (d) S24 (OV-275), (e) S27 (PEG), (f) S28 (PEG), (g) S4 (Carbowax 20M) and (h) S40 (Vaseline)]. 
this volatile compound. In general, $S 40$ (Vaseline) shows the highest results to the six volatile compounds, especially to undecane and Z-3-hexenyl acetate (Figure 3(h)). Finally, it is important to emphasize that sensors $S 4$ and $S 16$ (Figures 3(g) and 3(a)) cannot be used to analyze olive oil volatile compounds because they get the saturation when acetic acid (principal responsible of vinegary olive oil defect) [1,2,9] and Z-3-hexenyl acetate (related with olive oil green connotations) volatiles concentrations reach 20 ppm. Therefore, these sensors, S4 and S16, were rejected to constitute the QCM sensors array to detect volatile compounds with high olive oil sensory significance.

\subsection{Sensors Selection by Their Sensitivity Values}

As well, sensors curves represented in Figure 3 can now be used to evaluate the sensitivity of the QCM sensors. Sensitivities of the six sensors selected above, $S 20$ (OV-17), S24 (OV-275), S27 (PEG), S28 (PEG), S32 (Span 80 ) and $S 40$ (Vaseline), to individual VOCs tested of hexanal, acetic acid, Z-3-hexenyl acetate, undecane, 1-octen-3-ol and 2-butanone, have been measured. In this study, the average sensitivity $\left(S_{m}\right)$ of a QCM device coated with a sensing material may be defined as the mean value of the $n$ responses to different vapor concentrations of the same organic solvent:

$$
S_{m}=\frac{1}{n} \sum_{i=1}^{n} \frac{\Delta F_{i}}{c_{i}}
$$

where $\Delta F_{i}(\mathrm{~Hz})$ is the steady-sate frequency change of the sensor exposed to vapor concentration $c_{i}$ (ppm) of a given $i$ VOC tested. The results of each sensor have been normalized because each sensor oscillation frequency shift due to sensing film coating process (Table 2) has been subtracted to each sensor steady-state frequency change. These results have been reported in Table 3. Selected sensors show different sensitivities to volatile compounds. In general, high sensitivities of the sensors have been detected, compared with previous works $[6,15]$. Smaller sensitivities of the six sensors have been found for 2-butanone volatile compound. Sensor $S 40$ (Vaseline) shows the highest sensitivity values, especially for undecane and Z-3-hexenyl acetate, due to the low polarity values of Vaseline adsorbent and both volatile compounds. Maximum sensitivity value for 1-octen3-ol has been shown by sensor $S 32$ (Span 80), due to their similar polarity values [11]; according to Angerosa et al. [16] 1-octen-3-ol has the highest sensory significance to "mustiness/humidity" negative attribute, so this sensor $S 32$ could be very useful to detect olive oil samples with this sensory defect. Acetic acid can be discriminate by sensors $S 28$ (PEG), S27 (PEG) and S24 (OV-275) because they have highest sensitivity values for this volatile compound; moreover, acetic acid volatile compound has high sensory significance with olive oil "vinegary" negative attribute, so, these sensors could be used as detectors of olive oil samples with this defect. The greater sensitivity values for Z-3-hexenyl acetate are shown by sensors $S 40$ (Vaseline), $S 20$ (OV-17) and $S 32$ (Span 80), so they present the highest discrimination capacity for Z-3-hexenyl acetate; therefore, these sensors could be used to detect olive oil samples with green sensory connotations, e.g. at the beginning of the olive fruits harvesting time or olive oil varieties with green connotations, such as "Picual" or "Koroneiki", due to Z-3-hexenyl acetate present high significance with this positive olive oil sensory attribute $[3,17,18]$.

Ideally, we were looking for sensors which performances can be adjusted to Sauerbrey equation and show high discrimination capacity and sensitivity, together with a well differentiated pattern of response to the six volatile compounds defined, which means a potential capability to differentiate the corresponding aromas in olive oil. With this aim, we decided to select five sensors among those tested with the six substances. The choice of only five sensors for the array is due to the determination to simplify its construction, use and dates statistic analysis, and to reduce the cost of sensors preparation and maintain.

According to the criteria described above, the best sensors among those examined were $S 20$ (OV-17, $6 \mathrm{mg}$ ),

Table 3. Sensitivity values (Hz/ppm) of QCM sensors coated by OV-17 (S20), OV-275 (S24), PEG (S27 and S28), Span 80 (S32) and Vaseline (S40) to the organic volatile compounds tested.

\begin{tabular}{ccccccc}
\hline Sensor & Undecane & Acetic acid & 1-octen-3-ol & Z-3-hexenyl acetate & Hexanal & 2-butanone \\
\hline S20 & 4.831 & 2.315 & 2.894 & 16.783 & 3.487 & 0.992 \\
S24 & 0.521 & 16.881 & 3.425 & 6.681 & 4.619 & 1.942 \\
S27 & 1.633 & 11.450 & 6.017 & 6.407 & 1.895 & 0.487 \\
S28 & 7.165 & 21.515 & 4.571 & 3.979 & 2.190 & 0.373 \\
S32 & 18.787 & 12.456 & 23.265 & 16.583 & 14.646 & 1.458 \\
S40 & 70.088 & 5.642 & 17.477 & 56.398 & 11.315 & 1.290 \\
\hline
\end{tabular}


S24 (OV-275, 6 mg), S28 (PEG, 6 mg), S32 (Span 80, 6 $\mathrm{mg}$ ) and $S 40$ (Vaseline, $6 \mathrm{mg}$ ). They all show a reasonably good sensitivity, stability and discrimination capacity of the volatile compounds tested.

\section{Conclusion}

Forty quartz crystal microbalance sensors, each one coated with different concentrations of ten stationary phases materials for gas chromatography, have been evaluated as a sorption detectors for six volatile compounds with high olive oil sensory significance. Sensing films coating process, i.e. sensor construction process, presented in this study has been widely applied in previous works and guarantees applying a reproducible amount of coating material onto the quartz crystal resonator. The five coating materials selected, by Sauerbrey equation criteria, responses curves and sensitivity values of each sensor exposed to each volatile compound, have complementary polarities and high discrimination capacity of different volatile compounds. The QCM sensors array proposed will provide an incipient method, simple to use, low cost, easy portability and rapidity for classification of olive oil samples based on their volatile composition; as well to, it does not use solvents, it neither involves any pre-treatment of the sample. Moreover, the detection system presented herein is designed such that it would require minimal human supervision. The simplicity of the operation procedure is comparable to a commercially available UV-visible spectrometer and easier than other methods applied to detect volatile compounds, such as gas chromatography. Therefore, the quartz crystal microbalance sensors array system proposed could be a substitute technique to the analytical methods normally used for the analysis of the olive oil flavor.

\section{Acknowledgement}

This work was supported by a grant "Quickly sensory methods to olive oils characterization" (Andalusia Regional Government, Spain) and by the project INIA RTA 04-128, "Study and application of new techniques in virgin olive oil characterization during its production" (Spanish Science Ministry).

\section{References}

[1] “European Communities Regulation 640/2008,” Official Journal of European Communities, Legis Number 178, 2008, pp. 11-16.

[2] International Olive Oil Council, IOOC/T.20/Document 15/Rev. 2 (Organoleptic assessment of olive oil Resolution, September), Madrid, Spain, 2007.
[3] F. Angerosa, M. Servili, R. Selvaggini, A. Taticchi, S. Esposto and G. Montedoro, "Volatile Compounds in Virgin Olive Oil: Occurrence and Their Relationship with the Quality,” Journal of Chromatography A, Vol. 1054, No. 1, October 2004, pp. 17-31. doi:10.1016/j.chroma.2004.07.093

[4] M. E. Escuderos, M. Uceda, S. Sánchez and A. Jiménez, "Instrumental Technique Evolution for Olive Oil Sensory Analysis," European Journal of Lipid Sciences and Technology, Vol. 109, No. 5, May 2007, pp. 536-546. doi:10.1002/ejlt.200600239

[5] M. E. Escuderos, "QCM Piezoelectric Sensor to Olive Oil Sensory Characterization,” Ph.D. Thesis, University of Jaén, Spain, 2008.

[6] A. K. M. Shafiqul, Z. Ismail, M. N. Ahmad, B. Saad, A. R. Othman, A. Y. Md. Shakaff, A. Daud and Z. Ishak, "Transient Parameters of a Coated Quartz Crystal Microbalance Sensor for the Detection of Volatile Organic Compounds (VOCs)," Sensors and Actuators B, Vol. 109, No. 2, September 2005, pp. 238-243.

doi:10.1016/j.snb.2004.12.116

[7] X. Xu, H. Cang, C. Li, Z. K. Zhao and H. Li, "Quartz Crystal Microbalance Sensor Array for the Detection of Volatile Organic Compounds,” Talanta, Vol. 78, No. 3, May 2009, pp. 711-716. doi:10.1016/j.talanta.2008.12.031

[8] H.-C. Hana, Y.-R. Chang, W.-L. Hsu and C.-Y. Chen, "Application of Parylene-Coated Quartz Crystal Microbalance for On-line Real-Time detection of microbial populations,” Biosensors and Bioelectronics, Vol. 24, No. 6, February 2009, pp. 1543-1549. doi:10.1016/j.bios.2008.07.033

[9] M. T. Morales, G. Luna and R. Aparicio, "Comparative Study of Virgin Olive Oils Sensory Defects," Food Chemistry, Vol. 91, No. 2, June 2005, pp. 293-301. doi:10.1016/j.foodchem.2004.06.011

[10] A. Kanavouras, A. Kiritsakis and R. J. Hernández, "Comparative Study on Volatile Analysis of Extra Virgin Olive Oil by Dynamic Headspace and Solid Phase Micro-Extraction," Food Chemistry, Vol. 90, No. 1, March 2005, pp. 69-79. doi:10.1016/j.foodchem.2004.03.025

[11] R. Ni, X-B. Zhang, W. Liu, G. L. Shen and R.-Q. Yu, "Piezoelectric Quartz Crystal Sensor Array with Optimized Oscillator Circuit for Analysis of Organic Vapors Mixtures," Sensors and Actuators B, Vol. 88, No. 2, January 2003, pp. 198-204. doi:10.1016/S0925-4005(02)00345-3

[12] A. K. M. Shafiqul, Z. Ismail, B. Saad, A. R. Othman, M. N. Ahmad and A. Y. Md. Shakaff, "Correlation Studies between Electronic Nose Response and Headspace Volatiles of Eurycoma Longifolia Extracts," Sensors and Actuators B, Vol. 120, No. 1, December 2006, pp. 245-251. doi:10.1016/j.snb.2006.02.020

[13] A. K. M. Shafiqul, Z. Ismail, B. Saad, A. R. Othman, A. Y. Md. Shakaff, A. Daud and Z. Ishak, "Transient Parameters of Coated Quartz Crystal Microbalance Sensors for the Detection of Volatile Organic Compounds (VOCs)," Sensors and Actuators B, Vol. 109, No. 2, Sep- 
tember 2005, pp. 238-243.

doi:10.1016/j.snb.2004.12.116

[14] S. Muñoz, T. Nakamoto and T. Moriizumi, "Study of Quartz Crystal Microbalance Odor Sensing System for Apple and Banana Flavors," IEICE Transactions on Electronics, Vol. 85, No. 6, June 2002, pp. 1291-1297.

[15] A. S. Yuwono and P. S. Lammers, "Performance Test of a Sensor Array-Based Odor Detection Instrument," Journal of Science and Research Development, Vol. 3, 2004, pp. 9-25.

[16] F. Angerosa, B. Lanza and V. Marsilio, "Biogenesis of 'Fusty' Defect in Virgin Olive Oils,” Grasas y Aceites, Vol. 47, No. 3, 1996, pp. 142-150.

doi:10.3989/gya.1996.v47.i3.854
[17] J.F. Cavalli, X. Fernández, L. Lizzani-Cuvelier and A. M. Loiseau, "Comparison of Static Headspace, Headspace Solid Phase Microextraction, Headspace Sorptive Extraction, and Direct Techniques on Chemical Composition of French olive oils," Journal of Agricultural and Food Chemistry, Vol. 51, No. 26, December 2003, pp. 77097716. doi:10.1021/jf034834n

[18] W. Dhifi, F. Angerosa, A. Serraiocco, I. Oumar, I. Hamrouni and B. Marzouk, "Virgin Olive Oil Aroma: Characterization of Some Tunisian Cultivars," Food Chemistry, Vol. 93, No. 4, December 2005, pp. 697-701. doi:10.1016/j.foodchem.2004.12.036 